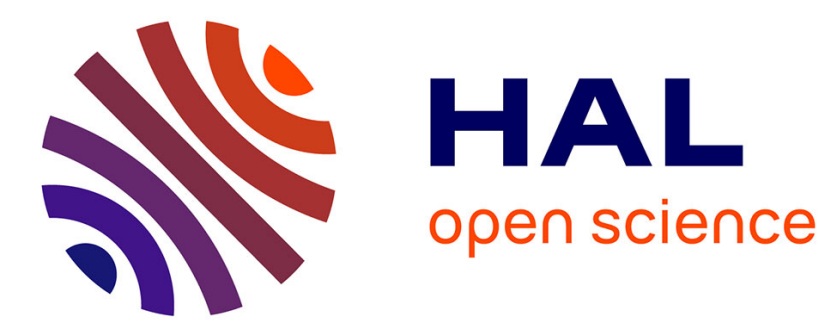

\title{
Les métamagnétiques ou substances antiferromagnétiques à champ seuil \\ Louis Néel
}

\section{To cite this version:}

Louis Néel. Les métamagnétiques ou substances antiferromagnétiques à champ seuil. Il Nuovo Cimento (1955-1965), 1957, 6 (3), pp.942-960. 10.1007/BF02834705 . hal-02887459

\section{HAL Id: hal-02887459 \\ https://hal.science/hal-02887459}

Submitted on 2 Jul 2020

HAL is a multi-disciplinary open access archive for the deposit and dissemination of scientific research documents, whether they are published or not. The documents may come from teaching and research institutions in France or abroad, or from public or private research centers.
L'archive ouverte pluridisciplinaire HAL, est destinée au dépôt et à la diffusion de documents scientifiques de niveau recherche, publiés ou non, émanant des établissements d'enseignement et de recherche français ou étrangers, des laboratoires publics ou privés. 


\title{
Les métamagnétiques ou substances antiferromagnétiques à champ seuil.
}

\author{
L. NÉEL \\ Institut Fourier, Université - Grenoble
}

\section{1. - Introduction et rappel de résultats antérieurs.}

Malgré son peu de rigueur, la théorie du champ moléculaire reste encore aujourd'hui le meilleur guide dans l'interprétation des propriétés magnétiques des substances antiferromagnétiques et ferrimagnétiques: nous voulons l'appliquer ici à une catégorie particulière de substances antiferromagnétiques, douées de propriétés spécifiques, et que nous nous proposons d'englober sous le nom de substances métamagnétiques, attribué déjà à certaines d'entre. elles par J. BECQUEREL ( ${ }^{1}$ ).

La théorie de l'antiferromagnétisme que nous avons proposée depuis assez longtemps $\left({ }^{2}\right)$ consiste à diviser le réseau des ions magnétiques en deux sousréseaux équivalents $A$ et $B$ dont nous désignons les aimantations par $\boldsymbol{M}_{a}$ et $\boldsymbol{M}_{b}$. Nous exprimons les différentes interactions d'échange ou de superéchange entre les ions par deux champs moléculaires $\boldsymbol{H}_{a}$ et $\boldsymbol{H}_{b}$, agissant respectivement sur les sous-réseaux $A$ et $B$ et donnés par les relations:

$$
\boldsymbol{H}_{\boldsymbol{a}}=n^{\prime} \boldsymbol{M}_{a}-n \boldsymbol{M}_{b} ; \quad \boldsymbol{H}_{b}=n^{\prime} \boldsymbol{M}_{b}-n \boldsymbol{M}_{a}
$$

$n$ et $n^{\prime}$ sont deux coefficients de champ moléculaire; le coefficient $n$ est essentiellement positif.

A haute température $\boldsymbol{M}_{a}=\boldsymbol{M}_{b}$ et la substance obéit à une loi de Curie-

(1) J. Becquerel: Journ. de Phys., 10, 10 (1939); Réunion Internationale de Magné. tisme (Strasbourg, 1939), tome I, p. 97.

$\left({ }^{2}\right)$ L. NǴ丶L: Ann. de Phys., 17, 5 (1932); Journ. de Phys., 3, 160 (1932); Ann. de Phys., 5, 232 (1936); Compt. Rend. Ac. Sci., 203, 304 (1936). 
Weiss classique; 1'aimantation $J$ s'écrit:

$$
J=\frac{C H}{T-\theta_{p}}
$$

où $U^{\prime}$ possède la valeur classique de la constante de Curie et où:

$$
\theta_{p}=\frac{1}{2} O\left(n^{\prime}-n\right)
$$

Au dessous d'une certaine température $T_{n}$, un ordre s'établit dans l'orientation des moments magnétiques et les deux sous-réseaux prennent des aimantations spontanées égales mais orientées en sens inverses l'une de l'autre: nous désignons par $\frac{1}{2} M$ la grandeur de l'aimantation spontanée de chaque sous-réseau. C'est une fonction de la température. Tout se passe comme si chaque sous-réseau était ferromagnétique pour son propre compte et prenait sous l'action d'un champ moléculaire positif $H_{m}=\left(n^{\prime}+n\right) M / 2$ une aimantation spontanée $M / 2$. Il en résulte que $T_{n}$ est donné par:

$$
T_{n}=\frac{1}{2} C\left(n^{\prime}+n\right)
$$

A la température $T=T_{n}$, la susceptibilité $s$ est donnée par:

$$
s=\frac{1}{n}
$$

\section{2. - Les différentes catégories de substances antiferromagnétiques.}

La valeur de $n$ étant donnée, celle de $n^{r}$ n'est pas quelconque: on conçoit en effet que si $n^{\prime}$ était négatif et grand en valeur absolue devant $n$, il devrait exister une autre décomposition du réseau principal en deux sous-réseaux correspondant à une énergie libre plus faible que celle de la décomposition primitivemente adoptée. Nous ne dirons rien de plus sur cette question très complexe de stabilité des sous-réseaux qui est liée à l'architecture du réseau cristallin et aux interactions entre un atome donné et ses voisins plus ou moins éloignés.

Les coefficients $n$ et $n^{\prime}$ peuvent se déterminer au moyen des formules (3) et (1), connaissant les valeurs de la température de Curie paramagnétique $\theta_{n}$ et de la température de transition $T_{n}$. Lorsqu'on effectue ces déterminations sur les quelques dizaines de substances antiferromagnétiques actuellement connues et étudiées, on s'aperçoit qu'il convient de les partager en trois groupes principaux. Un premier groupe comprenant des substances telles que FeO, 
MnO, etc., correspond à des valeurs de $n^{\prime}$ négatives comprises entre $-0.51 n$ et $-0.57 n$; les dispositions relatives des ions dérivent de celles a'un réseau cubique à faces centrées. Un deuxième groupe comprenant des substances comme $\mathrm{FeF}_{2}, \mathrm{CoF}_{2}$, ete., correspond à des valeurs de $n^{\prime}$ négatives comprises entre $-0.16 n$ et $-0.28 n$. Les dispositions relatives des ions dérivent de celles du cube centré: tous les proches voisins d'un atome d'un sous-réseau donné appartiennent à l'autre sous-réseau. Enfin, un troisième groupe comprenant des substances telles que $\mathrm{NiCl}_{2}, \mathrm{CoCl}_{2}$, etc., correspond à des valeurs de $n^{\prime}$ positives, souvent très grandes vis-à-vis de $n$. C'est ce troisième groupe de substances que nous nous proposons plus spécialement d'étudier dans ce mémoire.

\section{3. - Les couplages magnétocristallins.}

Pour compléter la description des propriétés d'une substance antiferromagnétique, il est nécessaire maintenant de tenir compte des couplages magnétocristallins. Ces couplages font apparaître dans l'expression de l'énergie totale un terme $E_{c}$ qui depend de l'orientation de la direction d'antiferromagnétisme par rapport aux axes du cristal. Supposons par exemple qu'il s'agisse d'une substance uniaxe, placée dans un champ magnétique nuì les aimantations $\boldsymbol{M}_{a}$ et $\boldsymbol{M}_{\boldsymbol{b}}$ des denx sous-réseaux sont rigoureusement antiparallèles et ce terme s'écrit en première approximation sous la forme:

$$
E_{o}=-K^{\prime} \cos ^{2} \theta
$$

où $\theta$ représente 1'angle avec l'axe de la direction d'antiferromagnétisme, c'est-àdire de la direction parallèle à $M_{a}$ et à $M_{b}$. Si paì exemple, la constante $K^{\prime}$ est positive, la direction d'antiferromagnétisme coïncide avec l'axe. Nous sommes très peu renseignés sur les valeurs de $K^{\prime}$; nous sommes cependant en droit d'admettre qu'elles sont du même ordre de grandeur que les constantes d'anisotropie des substances ferromagnétiques, c'est-à-dire de l'ordre de $10^{4}$ à $10^{6} \mathrm{erg} / \mathrm{g}$.

En l'absence de perturbations extérieures, les directions d'antiferromagnétisme occupent donc des directions privilégiées correspondant aux minima de $E_{c}$. On peut évidemment rapprocher ces directions privilégiées des directions privilégiées de l'aimantation spontanée, dans un corps ferromagnétique, mais il existe cependant une différence fort importante. En effet, l'application d'un champ $H$ correspond, dans une substance ferromagnétique, à un couple sur l'aimantation spontanée qui est de l'ordre de $M H$; al contraire, dans une substance antiferromagnétique, le couple qui agit sur la direction d'antiferromagnétisme est beaucoup plus petit, puisque de l'ordre de $H^{2} / n$. 
Il en résulte que les directions d'antiferromagnétisme sont probablement beaucoup plus difficiles à modifier que les directions d'aimantation spontanée.

\section{4. - Suseeptibilités parallèle et perpendieulaire.}

Du fait même de l'existence des directions privilégiées d'antiferromagnétisme, il convient de distinguer deux cas lorsqu'on applique un champ magnétique à un système antiferromagnétique: le cas où le champ appliqué est perpendiculaire à la direction privilégiée et le cas où le champ lui est parallèle.

Lorsque le champ est perpendiculaire à la direction privilégiée, le système prend une aimantation $J$ parallèle à $H$ donnée par:

$$
J=H / n
$$

la susceptibilité $J / H$ est indépendante de $H$ et égale à $1 / n$. On la désigne sous le nom de susceptibilité perpendiculaire et on la désigne par $s_{n}$.

Lorsque le champ $H$ est parallèle à la direction privilégiée d'antiferromagnétisme, la susceptibilité parallèle $s_{p}=J / H$ est toujours indépendante du champ, mais sa valeur est donnée par une expression plus compliquée:

$$
\frac{1}{s_{y}}=n-\frac{1}{2}\left(n+n^{\prime}\right) \frac{M}{T} \frac{\mathrm{d} T}{\mathrm{~d} M},
$$

où $\frac{1}{2} M$ représente l'aimantation spontanée de chacun des sous-réseaux, brièvement étudiée plus haut. A la température de transition $T_{n}$, le facteur $(M / T) \mathrm{d} T / \mathrm{d} M$ tend vers zéro, de sorte que $s_{\mathfrak{p}}$ tend vers $1 / n$, c'est-à-dire vers la valeur de $s_{n}$. Au contraire lorsque la température tend vers le zéro absolu, $(M / T) \mathrm{d} T / \mathrm{d} M$ tend vers l'infini, de sorte que $s_{p}$ tend vers zéro.

Ainsi, lorsque $n^{\prime}$ est de l'ordre de grandeur de $n$, la susceptibilité $s_{\vartheta}$ varie d'une manière relativement progressive de 0 à $1 / n$, lorsque $T$ varie du zéro absolu jusqu'à la température de transition $T_{n}$. Au contraire lorque $n^{\prime}$ est très grand devant $n$, la susceptibilité $s_{p}$ reste égale à zéro jusqu'à une température voisine de $T_{n}$, puis varie très rapidement poư atteindre la même valeur $1 / n$ pour $T=T_{n}$.

Lorsque le champ magnétique est parallèle à la direction privilégiée d'antiferromagnétisme, l'aimantation du système provient d'une augmentation de l'aimantation $\boldsymbol{M}_{a}$ du sous-réseau dont l'aimantation est dirigée dans le même sens que $\boldsymbol{H}$ et d'une diminution de $\boldsymbol{M}_{b}$, mais les orientations de $\boldsymbol{M}_{a}$ et de $\boldsymbol{M}_{b}$ restent invariables ainsi que la direction d'antiferromagnétisme. Au contraire, lorsque le champ est perpendiculaire à la direction d'antiferromagnétisme, $\boldsymbol{M}_{a}$ et $\boldsymbol{M}_{b}$ ne restent plus antiparallèles et tendent à s'aligner dans la direction 
du champ. On peut dans ce cas convenir d'appeler direction d'antiferromagnétisme la bissectrice extérieure de $\boldsymbol{M}_{a}$ et de $\boldsymbol{M}_{b}$ : la direction d'antiferromagnétisme reste alors invariable, quel que soit $\boldsymbol{H}$.

\section{5. - Le phénomène de découplage; le champ seuil.}

Une étude plus détaillée des phénomènes, dans le cas où le champ magnétique est parallèle à la direction d'antiferromagnétisme, montre qu'il existe une valeur critique du champ, appelée champ seuil, pour laquelle la direction d'antiferromagnétisme se découple brusquement de la direction privilégiée et lui devient perpendiculaire, avec variation discontinue de la susceptibilité qui de $s_{p}$ passe brusquement à la valeur $1 / n$, égale à $s_{n}$. Ce champ seuil est donné par:

$$
H_{s}^{2}=2 K^{\prime} n
$$

Nous avons signalé ce phénomène dès $1936\left(^{3}\right)$ et il a été retrouvé en 1952 , par C. J. GorTer et ses collaborateurs au cours d'une série de magnifiques travaux consacrés à l'étude des propriétés magnétiques de $\mathrm{CuCl}_{2}, 2 \mathrm{H}_{2} \mathrm{O}\left({ }^{4}\right)$.

Il convient ici de remarquer que dans le cas où $K^{\prime}$ est nul, e'est-à-dire en l'absence de couplage entre le réseau cristallin et la direction d'antiferromagnétisme, la direction d'antiferromagnétisme s'oriente toujours dans un sens perpendiculaire au champ appliqué et la susceptibilité conserve toujours une valeur égale à $1 / n$.

Dans la plupart des substances antiferromagnétiques, la valeur $H_{s} d u$ champ seuil est probablement très élevée, de sorte que la susceptibilité reste indépendante du champ jusqu'aux valeurs les plus élevées qu'il soit possible d'atteindre, mais il ne doit pas en être de même lorsque $n$ est petit: notamment lorsque le rapport $n^{\prime} / n$ est grand.

\section{6. - Les constantes $K_{0}$ et $K_{1}$ d'anisotropie d'un antiferromagnétique.}

Nous nous proposons maintenant de discuter l'influence de l'énergie magnétocristalline sur l'existence d'un champ seuil. Pour ne pas compliquer outre mesure la discussion, nous nous limiterons à un cas type simple: celui d'une substance uniaxe dans laquelle le rapport $u^{\prime} / n$ est grand. Dans ce cas, les modifications de l'état du système provoquées par l'action d'un champ extérieur

(3) L. NÉEL: Ann. de Phys., 5, 232 (1936).

(4) C. J. Gorter et H. HanntJes: Physica, 17, 285 (1952); J. Ubbink, J. A. Poulis et C. J. Gorter: Physica, 17, $213(1951) ; 18,361$ (1952). 
se réduisent à de simples changements d'orientation des aimantations spontanées $\boldsymbol{M}_{a}$ et $\boldsymbol{M}_{b}$ des deux sous-réseaux, ces aimantations conservant une grandeur égale à $\frac{1}{2} M$ qui ne dépend que de la température $T$. L'énergie totale du système est la somme de l'énergie d'échange de Weiss-Heisenberg, de l'énergie du systìme dans le champ extérieur $\boldsymbol{H}$ et de l'énergie magnétocristalline. La partie $E_{W}$ de l'énergie d'échange qui ne dépend que de l'angle de $\boldsymbol{M}_{a}$ avec $\boldsymbol{M}_{b}$ s'écrit:

$$
E_{w}=n \boldsymbol{M}_{a} \boldsymbol{M}_{b},
$$

tandis que l'énergie dans le champ extérieur est donnée par:

$$
E_{h}=-\boldsymbol{H}\left(\boldsymbol{M}_{a}+\boldsymbol{M}_{b}\right)
$$

En ce qui concerne maintenant l'énergie magnétocristalline $E_{c}$, l'état du système est défini par les angles $\theta$ et $\theta^{\prime}$ de $\boldsymbol{M}_{a}$ et de $\boldsymbol{M}_{b}$ avec l'axe ainsi que par l'angle $\varphi$ que font entre eux les plans méridiens contenant $\boldsymbol{M}_{a}$ et $\boldsymbol{M}_{b}$ : Compte-tenu du fait que dans l'expression générale de $E_{c}$ il existe des termes qui ne dépendent que de l'angle de $\boldsymbol{M}_{a}$ avec $\boldsymbol{M}_{b}$ et que ces termes, possédant ainsi un caractère isotrope, peuvent être intégrés dans les termes d'échange en modifiant convenablement la valeur du coefficient $n$, on démontre que l'expression générale de l'énergie magnétocristalline $E_{\mathrm{c}}$ d'une substance antiferromagnétique uniaxe, limitée aux termes du second degré par rapport aux cosinus directeurs de $\boldsymbol{M}_{a}$ et de $\boldsymbol{M}_{v}$, ne dépend que de deux constantes $\boldsymbol{K}_{0}$ et $K_{1}$ et s'écrit $\left({ }^{5}\right)$ :

$$
E=-\frac{1}{2} K_{0}\left(\cos ^{2} \theta+\cos ^{2} \theta^{\prime}\right)-K_{1} \cos \theta \cos \theta^{\prime}
$$

\section{7. - Etats d'équilibre du système.}

Dans un champ magnétique extérieur nul, l'état d'équilibre du système s'obtient en déterminant le minimum de $E_{w}+E_{c}$; par raison de symétrie, il correspond toujours à $\varphi=0$. Trois états sont possibles: 2 états antiferromagnétiques et un état ferromagnétique. Dans les deux états antiferromagnétiques, $(A p)$ et $(A n)$, les directions d'antiferromagnétisme sont respectivement parallèle et normale à l'axe. Dans l'état ferromagnétique (Fp), la direction privilégiée de l'aimantation spontanée est parallèle à l'axe du cristal.

Pour exprimer d'une manière simple les résultats, nous atiliserons deux variables réduites auxiliaires $r$ et $r^{\prime}$, dont les dimensions sont celles d'un nombre,

(5) L. NÉeL: Compt. Rend. Ae. Sci., 242, 1549 (1956). 
définies, par les relations suivantes:

$$
r=\frac{2\left(K_{0}+K_{1}\right)}{n M^{2}}, r^{\prime}=\frac{2\left(K_{0}-K_{1}\right)}{n M^{2}}
$$

On trouve alors $\left(^{5}\right)$ que lorsque $r^{\prime}$ est négatif, le système prend l'état (An) lorsque $r$ est inférieur à 1 et l'état (Fp) lorsque $r$ est supérieur à 1 . Lorsque $r^{\prime}$ est positif, le système prend l'état (Ap) lorsque $r-r^{\prime}$ est inférieur à 1 et

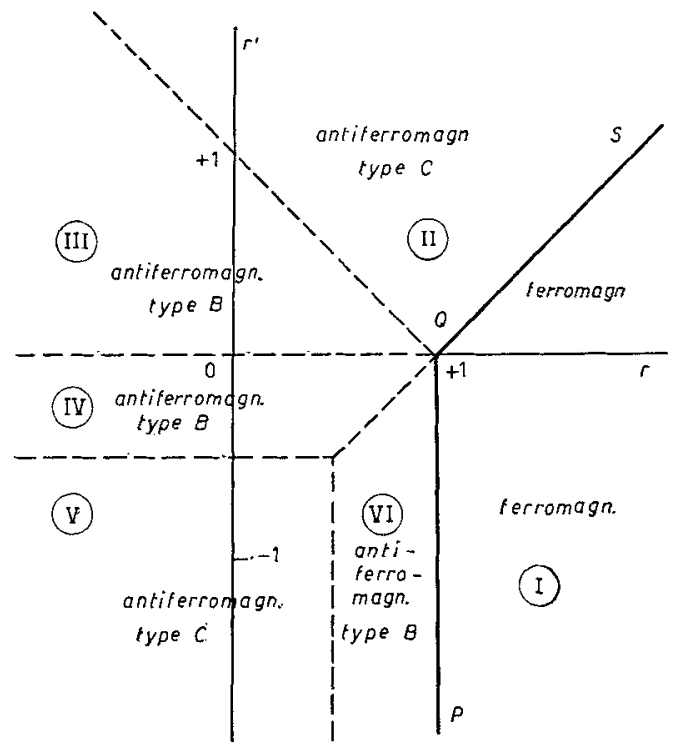
l'état (Fp) lorsque $r-r^{\prime}$ est supérieur à 1 .

En portant sur un graphique (Fig. 1) des points représentatifs, de coordonnées rectangulaires $r$ et $r^{\prime}$, on pent ainsi diviser le plan $\left(r, r^{\prime}\right)$ en deux régions par la ligne brisée $P Q S$ : à droite la région ferromagnétique, à gauche la région antiferromagnétique.

Fig. 1. - Diagramme d'état dans le plan $\left(r, r^{\prime}\right)$ (antiferromagnétisme ou ferromagnétisme). Quand $r^{\prime}$ est positif, l'axe cristallin et la direction d'antiferromagnétisme sont parallèles; ils sont perpendiculaires quand $r^{\prime}$ est négatif.

\section{8. - Action d'un champ magnétique extérieur.}

Il importe maintenant de compléter l'étude de la région antiferromagnétique par la détermination de l'aimantation obtenue en soumettant le système à un champ extérieur $H$. Il suffit pour cela d'ajonter à l'énergie du système le terme $E_{h}$ donné plus haut par la relation (11) et d'écrire la condition d'énergie minimale. Les calculs sont toujours très élémentaires aussi nous nous contenterons d'en énoncer seulement les résultats, qui sous une forme partielle avaient déjà fait l'objet d'une publication antérieure $\left(^{6}\right)$. Pour abréger l'écriture, nous exprimerons en outre les champs magnétiques $H$ par l'intermédiaire d'une variable

${ }^{6}$ ) L. NÉEt: $X^{e}$ Conseil de Physique Solvay (Bruxelles, Septembre 1954). (Les électrons dans les métaux, R. SToops, éditenr, Bruxelles, 1955, p. 251). 
réduite:

$$
h=\frac{2 H}{n M}
$$

On constate d'abord qu'en appliquant le champ magnétique $H$ dans une direction perpendiculaire à la direction d'antiferromagnétisme l'aimantation reste proportionnelle à $H$ jusqu'à ce que la saturation $M$ soit atteinte. La susceptibilité $s_{n}$ conserve donc une valeur constante jusqu'à ce que le champ réduit $h$ atteigne une valeur $h_{1}$ et on obtient une courbe d'aimantation du type $A$ (Fig. 2). L'inverse $1 / s_{n}$ de la susceptibilité perpendiculaire prend la valeur $n\left(1+r^{\prime}\right)$ lorsque $r^{\prime}$ est positif et la valeur $n(1-r)$ lorsque $r^{\prime}$ est négatif (dans le premier cas la direction d'antiferromagnétisme est parallèle à l'axe, dans le second cas, elle lui est perpendiculaire).

Ainsi, dans les deux cas,

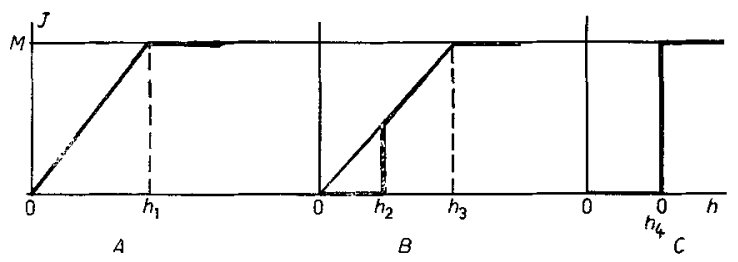

Fig. 2. nous obtenons une valeur de la susceptibilité $s_{n}$ nettement différente de la valeur conventionnelle égale à $1 / n$. Cette observation a son intérêt car, comme nous le préciserons plus loin, la variable $r^{\prime}$ est susceptible de varier d'une manière extrêmement importante avec la température, beaucoup plus que n. On pourrait pent-être expliquer ainsi à partir de cette remarque les variations anormales de $s_{n}$ qui ont parfois été observées.

Les lois de l'aimantation du système dans un champ parallèle à la direction d'antiferromagnétisme sont beaucoup plus compliquées que dans le cas précédent, comme conséquence de l'existence nécessaire d'un champ seuil. En effet, dans la phase initiale de l'aimantation la direction d'antiferromagnétisme, définie comme la bissectrice extérieure de $\boldsymbol{M}_{a}$ et $\boldsymbol{M}_{b}$, est parallèle á la direction du champ, tandis que dans la phase finale, au moment où $\boldsymbol{M}_{a}$ et $\boldsymbol{M}_{b}$ sont devenus parallèles, elle est perpendiculaire à la direction du champ: il y a donc eu discontinuité pour un certain champ seuil $h_{s}$. Deux types $B$ et $C$ de loi d'aimantation se rencontrent: un type $B$ dans lequel l'aimantation reste nulle jusqu'à ce que le champ atteigne une valeur seuil $h_{2}$ (Fig. 2) pour laquelle l'aimantation varie d'une manière discontinue et atteint une valeur inférieure à l'aimantation à saturation $M$. Ensuite, l'aimantation reste proportionnelle au champ, avec un rapport constant $J / H=s_{p}$, puis atteint la saturation pour $h=h_{3}$. Dans le type $C$, la discontinuité qui se produit pour le champ seuil $h_{1}$ est telle que la saturation $M$ est atteinte d'emblée.

Dans cette description, nous supposons que la susceptibilité est nulle dans la phase initiale, au-dessous du champ seuil. Nous rappelons, comme cela a 
été précisé plus haut (cf. Sect. 4), que cette hypothèse est correcte jusqu'à une température très proche de la température de transition $T_{n}$, puisque nous nous sommes placés par hypothèse dans le cas où $n^{\prime}$ est grand vis-à-vis de $n$.

Nous avons déterminé, en fonction de $r$ et de $r^{\prime}$, les types de lois d'aimantation rencontrés ainsi que les valeurs des champs critiques $h_{2}, h_{3}, h_{4}$.

Le plan $\left(r, r^{\prime}\right)$ est divisé, comme le montre la Fig. 1, en 6 régions par les 6 droites $r^{\prime}=0 ; r=1 ; r^{\prime}=-\frac{1}{2} ; r=\frac{1}{2} ; r-r^{\prime}=1 ; r+r^{\prime}=1$. Le Tableau I rassemble le résultat des calculs.

Lorsqu'au lieu d'étudier un monocristal on étudie une poudre agglomérée, composée de cristallites orientés au hasard, la susceptibilité moyenne $s_{m}$ s'obtient en première approximation par la relation:

$$
s_{m}=2 s_{n}+s_{p} .
$$

Cette formule n'est pas rigoureuse pon deux raisons: elle néglige les interactions magnétiques entre les cristallites et elle néglige aussi la rotation de lar direction d'antiferromagnétisme qui se produit lorsque le champ est appliqué obliquement par rapport à la direction d'antiferromagnétisme (cette rotation. est de l'ordre de $h^{2}$, tandis que la rotation de $\boldsymbol{M}_{a}$ ou de $\boldsymbol{M}_{b}$ est de l'ordre de h). A cause de sa simplicité, nous utiliserons néanmoins la formule (15): elle montre que la présence d'un champ seuil se manifeste, dans le cas d'une poudre, par une variation beaucoup plus rapide de l'aimantation en fonction de $h$.

\section{9. - Hystérésis paramagnétique.}

Les résuitats qui ont été résumés dans le Tableau I ont été obtenus en supposant que le système prend toujours la configuration qui correspond à l'énergie la plus basse. En réalité, de faux équilibres sont possibles; l'analyse des phénomènes, relatifs au type $B$ d'aimantation par exemple, montre (6) qu'à champ magnétique croissant le découplage de la direction d'antiferromagnétisme ne doit se produire que pour un champ $h_{2}^{\prime}$ supérieur à $h_{2}$; à champ décroissant, le retour à sà position initiale de la direction d'antiferromagnétisme ne se produit que pour un champ $h_{2}^{\prime \prime}$ inférieur à $h_{2}$. On obtient ainsi de véritables phénomènes d'hystérésis.

Du point de vue expérimental, nous ne conmaissons rien de précis à ce sujet. Nous n'insisterons done pas davantage d'autant plus qu'il est fort possible qu'il existe un mécanisme qui permette au système de prendre automatiquement la configuration d'énergie minimale. On sait qu'un tel mécanisme, à savvoir le déplacement des parois de Bloch, permet, dans un corps ferromagné- 


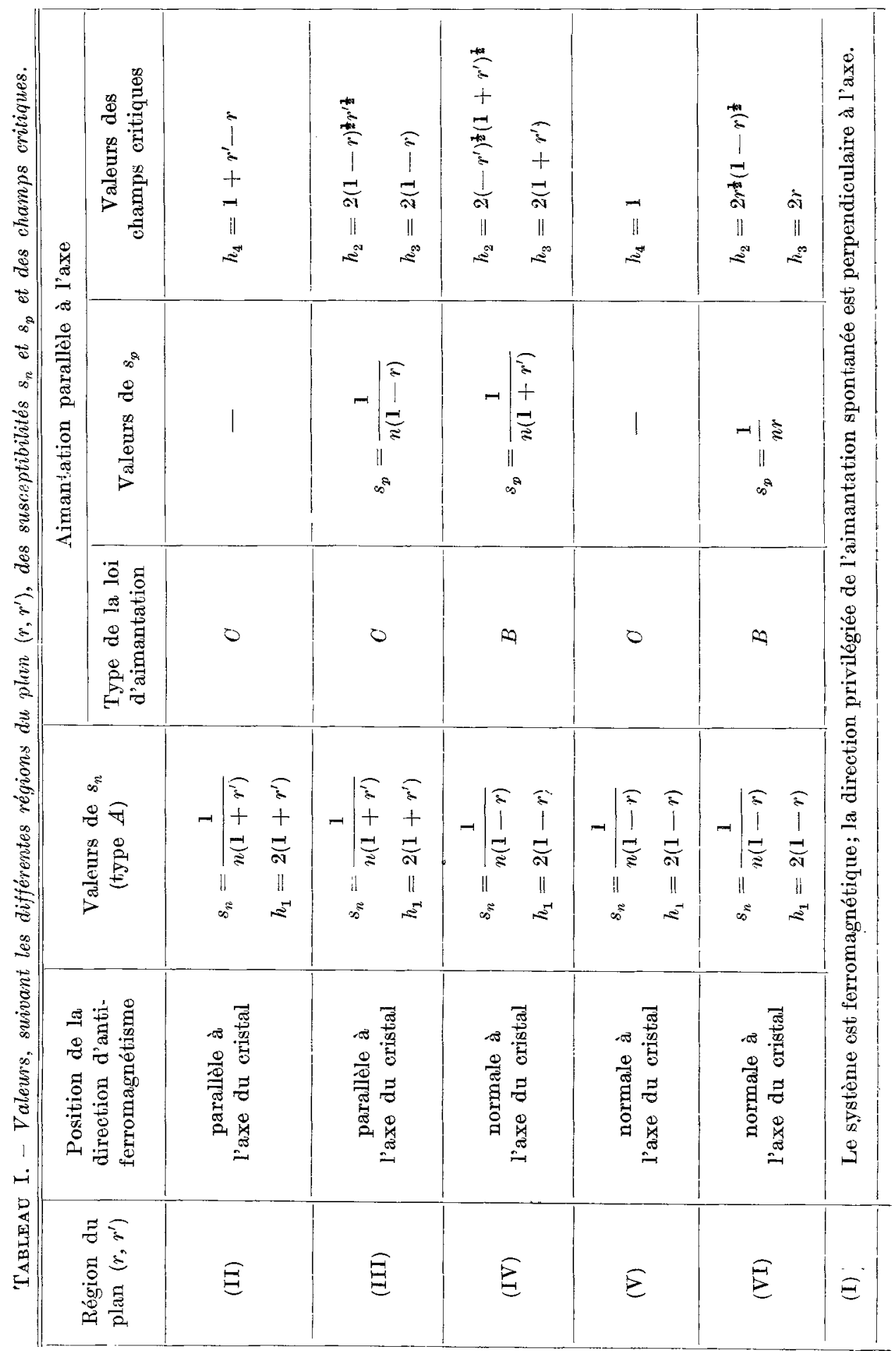


tique, le choix, entre deux phases aimantées en sens inverse, de la phase d'énergie minimale pour un champ appliqué sensiblement nul, tandis que le passage réversible d'une phase à l'autre exigerait des champs magnétiques très élevés. On peut de même, dans une substance antiferromagnétique, supposer qu'il existe toujours, mais en proportions variables, deux phases dont l'une correspond à une direction d'antiferromagnétisme parallèle à l'axe, l'autre à une direction perpendiculaire et imaginer que les changements de proportion entre les phases s'effectuent par le déplacement de la paroi qui les sépare.

\section{0. - Applieation aux chlorures anhydres.}

Les considérations développées plus haut s'appliquent immédiatement aux chlorures anhydres, $\mathrm{NiCl}_{2}, \mathrm{CoCl}_{2}, \mathrm{FeCl}_{2}$, comme nous avons en l'occasion de le montrer récemment ( $\left.{ }^{6}\right)$.

Les chlorures $\mathrm{NiCl}_{2}, \mathrm{CoCl}_{2}$ appartiennent très probablement au type $B$, comme le montre le fait que la susceptibilité dans des champs de l'ordre de 1000 Oe est nettement plus faible, de l'ordre des deux tiers, que la susceptibilité dans 10000 Oe: c'est à quoi il faut s'attendre, dans le cas d'une poudre, lorsqu'il existe un champ seuil de valeur intermédiaire. Lies données expérimentales ne permettent pas de déterminer les 4 constantes $n^{\prime}, n, r$ et $r^{\prime}$ car il existe seulement deux bonnes données, la température de transition $T_{n}$ et la susceptibilité dans les champs forts pratiquement égale à $s_{n}$, et une donnée très mal connue: la valeur du champ seuil. $\mathrm{Si}$ on suppose, tout gratuitement d'ailleurs, $r=r^{\prime}$, on obtient, en rapportant les aimantations à 1 gramme, les valeurs indiquées dans le Tableau II. Les point représentatifs seraient ainsi situés dans la région (III) du plan $\left(r, r^{\prime}\right)$, sur la première bissectrice.

TABLEAU II.

\begin{tabular}{|c|c|c|c|}
\hline \hline Substance & $\mathrm{NiCl}_{2}$ & $\mathrm{CoCl}_{2}$ & $\mathrm{FeCl}_{2}$ \\
\hline$n^{\prime}$ & 11000 & 3000 & 3400 \\
$n$ & 1300 & 340 & 1.30 \\
$r$ & 0.0016 & 0.054 & 0.5 \\
\hline
\end{tabular}

Pour $\mathrm{FeCl}_{2}$, l'amplitude de Ia discontinuité correspondant au champ seuil devient extrêmement importante, de l'ordre de grandeur de l'aimantation à saturation: on a affaire au type $C$ (Fig. 2) et le point représentatif passe dans la région (II) du plan $\left(r, r^{r}\right)$.

Pour les trois chlorures, le rapport $n^{\prime} / n$ est élevé: 10 au moins. La susceptibilité initiale varie comme l'inverse de $n(1+r)$; quand $r$ est petit, l'expérience 
montre que la susceptibilité est pratiquement indépendante de la température, d'où on déduit que $n$ est aussi pratiquement indépendant de la température. C'est un résultat tout à fait logique, puisque $n$ représente des interactions d'échange. Au contraire, la susceptibilité initiale de $\mathrm{FeCl}_{2}$ varie beaucoup avec $T$ : il faut certainement voir là l'influence de la variation thermique de $r$ qui, étant légal à 0.5 n'est plus négligeable devant l'unité.

\section{1. - Application à $\mathrm{MnAu}_{2}$.}

A. Meyer et P. Taglang ont récemment montré ( $\left.{ }^{7}\right)$ que la susceptibilité magnétique du composé métallique $\mathbf{M n A u}_{2}$ restait indépendante de la température de $100^{\circ} \mathrm{K}$ à $300^{\circ} \mathrm{K}$ et du champ magnétique jusqu'à $10000 \mathrm{Oe}$ environ. Au voisinage de cette valeur du champ, l'aimantation $J_{s}$ varie d'une manière beaucoup plus rapide puis tend ensuite vers la saturation selon une loi d'approche de la forme:

$$
J=J_{s}\left(1-\frac{b}{H^{2}}\right) \text {. }
$$

Comme nous l'avons déjà exposé (5) e'est exactement le comportement qu'il faut attendre de la part d'ume substance antiferromagnétique, caractérisée par nne loi d'aimantation du type $C$ et un champ seuil voisin de 10000 Oe. On peut supposer que le point représentatif soit situé dans la région (II) du plan $\left(r, r^{\prime}\right)$.

De la valeur de la susceptibilité initiale $s_{m}=3.76 \cdot 10^{-4}$, mesurée sur un polycristal, on tire:

$$
n \cdot\left(1+r^{\prime}\right)=1770
$$

de $100^{\circ} \mathrm{K}$ à $300^{\circ} \mathrm{K}$. De la détermination du champ seuil $H_{s}$ et de la valeur de l'aimantation à saturation $M$, on déduit que dans le même intervalle de température $H_{s} / M$ reste voisin de 270, d'où:

$$
n\left(1+r^{\prime}-r\right)=540,
$$

mais cela ne suffit pas encore pour déterminer $n, r^{\prime}$ et $r$.

On peut remarquer cependant que dans le cas du fer et du nickel, les mieux étudiés des ferromagnétiques, le rapport $K / M^{2}$ de la constante d'aniso-

(7) A. Merer et P. Taglang: Compt. Rend. Ac. Sci., 239, 961, 1611 (1954); Journ. de Phys. Rad., 17, (1956), sous presse. 
tropie au carré de l'aimantation spontanée tend vers zéro au point de Curie. Par analogie, il est logique d'admettre que dans les substances antiferromagnétiques $r$ et $r^{\prime}$ tendent aussi tous les deux vers zéro au point de transition $T_{n}$. Il en résulte alors que la susceptibilité pour $T=T_{n}$ est simplement égale à $1 / n$. Les mesures de A. MEYer et P. TAgLANG montrent que la susceptibilité de $\mathrm{MnAu}_{2}$ passe, au voisinage de $T_{n}=370^{\circ} \mathrm{K}$, par un maximum aigu égal à $1.1 \cdot 10^{-3}$ qui correspond à $n=900$ environ.

En admettant alors que $n$ ne varie pas avec $T$, on déduit alors des deux formules (17) et (18) que dans le domaine de température compris entre $100^{\circ} \mathrm{K}$ et $300^{\circ} \mathrm{K}, r$ et $r^{\prime}$ sont respectivement voisins de 1.4 et de 1.0.

D'autre part, on déduit $n^{\prime}-n$ de la valeur du point de Curie paramagnétique $\left(\theta_{p}=451^{\circ} \mathrm{K}\right)$ et de la constante de Curie spécifique $\left(C==7.12 \cdot 10^{-3}\right)$ en utilisant la formule (3), ce qui donne $x^{\prime}=128000$ environ. Le rapport $n^{\prime} / n=142$ est ici particulièrement grand. Cette grandeur du rapport $n^{\prime} / n$, jointe au fait que $r$ et $r^{\prime}$ passent de la valeur 0 aux valeurs 1.4 et 1.0 quand la température s'abaisse de $370^{\circ} \mathrm{K}$ à $300^{\circ} \mathrm{K}$, permet de comprendre pourquoi le maximum de la susceptibilité initiale à $T=T_{n}$ est aussi aigu. Un autre fait confirme l'exactitude de notre interprétation: lorsque le champ appliqué est suffisamment élevé $\left[h>2\left(1+r^{2}\right)\right]$, les deux aimantations spontanées $M_{a}$ et $M_{b}$ coïncident dans chacun de tous les cristallites et tout se passe comme si nous avions affaire à une substance ferromagnétique uniaxe et d'énergie d'anisotropie égale à - $\left(K_{0}+K_{1}\right) \cos ^{2} \theta$. La loi d'approche à la saturation est alors du type (16), avec:

$$
b=\frac{4 K^{2}}{n M^{2}}=\frac{n^{2} r^{2} M^{2}}{15}
$$

D'après les mesures de Meyer et TAGLAN'G, $b / M^{2}$ reste, de 100 à $300^{\circ} \mathrm{K}$, voisin de $0.64 \cdot 10^{5}$ d'où l'on déduit $n r=980$, alors qu'on déduit $n r=1260$ des données antérieurement admises: c'est un résultat très satisfaisant eu égard au caractère extrêmement schématique de la théorie.

\section{2. - La variation thermique de $r$ et de $r^{\prime}$ : transition entre ferromagnétisme et antiferromagnétisme.}

Nous avons déjà précisé plus haut les raisons expérimentales qui amènent à penser que $r$ et $r^{\prime}$ tendent vers zéro à la température $T_{n}$ de disparition de l'aimantation spontanée. Ces arguments sont d'ailleurs confirmés par les résultats d'une théorie due à Arulov ( ${ }^{8}$ ) selon laquelle la constante d'aniso-

(8) N. Akulov: Zeits. f. Phys., 100, 197 (1936). 
tropie d'un corps cubique ferromagnétique varierait au voisinage du point de Curie comme la dixième puissance de l'aimantation spontanée d'où il résulte que $r$ et $r^{\prime}$ varieraient comme $M^{8}$ et seraient nuls pour $T=T_{n}$. Nous considérerons désormais ce point comme acquis.

Loin du point de transition, les constantes d'anisotropie sont quelquefois proportionnelles au carré de l'aimantation spontanée: $r$ et $r^{\prime}$ sont alors constants, comme cela se passe pour $\mathrm{MnAu}_{2}$ au-dessous de $300^{\circ} \mathrm{K}$. Mais il existe beaucoup d'autres cas où la constante d'anisotropie varie d'une manière très importante et beaucoup plus compliquée par exemple dans des substances hexagonales comme Co ou MnBi. Dans le cobalt, K diminue de $4.7 \cdot 10^{7} \mathrm{erg} / \mathrm{mole}$ à zéro, lorsque la température passe de $-200^{\circ} \mathrm{C}$ à $+200^{\circ} \mathrm{C}$, dans un domaine où la diminution de $M^{2}$ est seulement de l'ordre de $5 \%$. De même, entre $84^{\circ} \mathrm{K}$ et $300^{\circ} \mathrm{K}$, la constante $K$ du composé $\mathrm{MnBi}$ augmente de 0 à $3.5 \cdot 10^{8} \mathrm{erg} / \mathrm{mole}$ tandis que $M^{2}$ diminue légèrement $\left(^{9}\right)$.

Finalement, nous arrivons à la conclusion $\left.{ }^{(10}\right)$ que le point représentatif $R$ de l'état du système antiferromagnétique se déplace dans le plan $\left(r, r^{\prime}\right)$ en fonction de la température pour aboutir à l'origine, à $T=T_{n}$. Il peut arriver notamment que $R$, situé initialement à basse température dans la partie de la région (I) où $r^{\prime}$ est positif (Fig. 1), se déplace, franchisse à une certaine température $T_{a}$ la frontière $Q S$ entre les régions (I) et (II), franchisse ensuite à une température $T_{b}$ la frontière entre les régions (II) et (III) pour aboutir à l'origine 0 , quand $T=T_{n}$. Nous serions donc ainsi en présence d'une substance qui est ferromagnétique à basse température et qui devient ensuite antiferromagnétique à $T=T_{a}$. L'antiferromagnétisme appartiendrait au type $C$ pour $T<T_{b}$ et au type $B$ pour $T_{b}<T<T_{n}$.

Il résulte des des considérations développées plus haut que, dans la région (II) $\left(T_{a}<T<T_{b}\right)$, le champ seuil $H_{s}$ et l'aimantation spontanée $M$ sont liées par la relation:

$$
\frac{H_{s}}{M}=\frac{1}{2} n\left(1+r^{\prime}-r\right) .
$$

On remarque d'abord que le champ seuil $H_{s}$ tend vers zéro quand $T$ tend vers $T_{a}$ puisque l'équation de la frontière $Q S$ entre les régions (I) et (II) est précisément $1+r^{\prime}-r=0$. $\mathrm{Il}$ en résulte qu'en portant $H_{s} / M$ en fonction de $T$ on obtient une courbe qui d'une part tend vers 0 lorsque $T$ tend vers $T_{a}$ et qui d'autre part, extrapolée vers $T=T_{n}$, tend vers $\frac{1}{2} n$. Il faut bien noter que cette extrapolation doit se faire à travers la région (II) $\left(T_{b}<T<T_{n}\right)$, à l'intérieur de laquelle il existe bien encore un champ seuil, mais donné par une formule différente: $H_{s} / M=n(1-r)^{\frac{\mathbb{f}}{{ }^{\prime}} r^{\prime}}$.

$\left({ }^{9}\right)$ C. Gumi,aud: Thìse (Strasbourg, 1943).

${ }^{(10)}$ L. NÉEL : Compt. Rend. Ac. Sci., 242, 1549, 1824 (1956). 


\section{3. - Autre type de transition entre ferromagnétisme et antiferromagnétisme.}

Dans le type de transition étudié plus haut, $r^{\prime}$ est positif. On peut concevoir aussi un autre type de transition correspondant au cas où $r^{\prime}$ est négatif. Le point représentatif $R^{\prime}$, situé initialement à basse température dans. la partie inférieure de la région (I) du plan $\left(r, r^{\prime}\right)$, se déplace, franchit à une certaine température $T_{n}$ la frontière $P Q$ entre les régions (I) et (VI), traverse ensuite les régions (VI), (IV) et éventuellement la région (V) pour aboutir finalement à l'origine 0 .

Ce deuxième type de transition entre ferromagnétisme et antiferromagnétisme est bien différent du premier. En effet, dans la région (VI), la loi d'aimantation est du type $B$ et le champ seuil, qui est donné par la relation. $H_{s} / M=n(1-r)^{\frac{1}{2}} r^{\frac{1}{2}}$, tend également vers zéro quand $T=T_{n}$ puisque l'équation de la frontière $P Q$ est $r=1$. Mais il y a une différence essentielle: dans. ce deuxième type, la discontinuité d'aimantation qui est associée au champ. seuil tend verso zéro quand $T$ tend vers $T_{a}$ tandis que dans le type précédent. l'amplitude de la discontinuité d'aimantation restait égale à $M$. Il doit done être relativement facile de distinguer les deux cas du point de vue expérimental.

\section{4. - Autres mécanismes de transition.}

L'étude du rôle des couplages magnétocristallins sur la stabilité des différentes configurations possibles vient de nous montrer que la variation thermique des constantes d'anisotropie pouvait donner naissance, pour une substance donnée, à une transition d'un état ferromagnétique à un état antiferromagnétique. Ce mécanisme de transition n'est naturellement pas le seul possible. J. S. SmaRT ( ${ }^{11}$ ) a déjà discuté les différentes manières suivant lesquelles. l'ordre pouvait s'établir dans une substance magnétique, pour différents réseaux cristallins, en fonction de la valeur des interactions d'écbange ou de superéchange entre un atome et ses voisins plus ou moins proches. Si ces interactions varient, e'est-à-dire en somme si $n$ et $n^{\prime}$ sont des fonctions de $T$, il peut se produire des transitions de l'état ferro- à l'état antiferro-magnétique.

La théorie de Smart, appliquée par cet auteur au cas de MnBi et de MnAs, n'invoque que la variation thermique des interactions isotropes d'échange ou de superéchange: elle ignore complètement le rôle des constantes d'anisotropie et l'existence du champ souil. Elle ne conduit done à aucune relation entre la valeur du champ seuil et la température de transition. Il n'est pas certain

(11) J. S. SmaRt: Naval Res. Rep., no. 2113 (Juillet 1951), U.S. Naval Ordnance Lab.; Phys. Rev., 90, 55 (1953). 
d'autre part que la variation thermique de $n$ et de $n^{\prime}$ soit suffisante pour faire apparaître de tels phénomènes: au surplus on a le moyen de savoir si les coefficients de champ moléculaire dépendent de la température. Nous avons en effet montré autrefois $\left(^{12}\right)$ que dans une substance présentant un paramagnétisme du type Langevin-Weiss (c'est le cas des substances antiferromagnétiques au-dessus de leur température $T_{n}$ de transition) la constante de Curie n'était égale à sa valeur théorique, relative à un paramagnétisme pur sans champ moléculaire, que dans les cas où les coefficients de champ moléculaire étaient indépendants de la température. Dans les autres cas, il y a un terme correctif.

\section{5. - Application à l'interprétation des propriétés magnétiques du dysprosium.}

Les propriétés magnétiques du dysprosinm métallique ont fait l'objet, ces. dernières années, d'études détaillées de F. Tromse $\left({ }^{13}\right)$ et de F. J. Elliots, S. Legvold et F. H. Spedding $\left({ }^{14}\right)$ elles sont très complexes et fort curieuses. Dans un domaine $A$ correspondant aux températures inférieures à $90^{\circ} \mathrm{K}$, le dysprosium se comporte comme un ferromagnétique classique. Dans un domaine $B\left(90^{\circ} \mathrm{K}<T<160^{\circ} \mathrm{K}\right)$, les isothermes d'aimantation présentent l'allure schématique suivante: l'aimantation commence par varier à peu près proportionnellement au champ jusqu'à un certain champ senil $H_{s}$, pour lequel s'amorce une variation beaucoup plus rapide de l'aimantation qui se termine finalement par une approche à la saturation en $1 / H^{2}$. Ces isothermes présentent une analogie très irappante avec celles de $\mathrm{MnAu}_{2}$, mais tandis que le champ seuil de $\mathrm{MnAu}_{2}$ varie assez peu avec la température celui de Dy varie au contraire beaucoup: de 2500 à 10000 Oe, quand $T$ passe de $103^{\circ} \mathrm{K}$ à $153^{\circ} \mathrm{K}$.

Plus loin, dans une région $O$ située au-dessus de $160^{\circ} \mathrm{K}$, le dysprosium devient paramagnétique avec une susceptibilité spécifique qui commence par croître avec la température, passe à $178^{\circ} \mathrm{K}$ par un maximum aigu voisin de $4 \cdot 10^{-3}$, puis décroît quand la température augmente davantage, en obéissant à une loi de Curie-Weiss définie par une constante de Curie spécifique $C=0.087$ et un point de Curie paramagnétique $\theta_{p}=157^{\circ} \mathrm{K}$.

Il s'agit certainement dans cette région $C$ d'un antiferromagnétisme de point de transition $T_{n}=178^{\circ} \mathrm{K}$. On obtient le coefficient $n$ par la relation. $n=1 / s$, où $s$ est la susceptibilité pour $T=T_{n}$, soit $4 \cdot 10^{-3}$, ce qui donne $n=250$. On a d'autre part $n^{\prime}-n=2 \theta_{p} / C$, d'ou $n^{\prime}-n=3600$ et finalement $n^{\prime}=4100$. Le rapport $n^{\prime} / n$ est ainsi assez grand, voisin de 16, de sorte que le dysprosium

(12) L. NÉes: Ann. de Phys., II e Série, 8, 237 (1937).

(13) F. Trombe: Journ. Rech. CNRS, no. 23 (Juin 1953), p. 61.

(14) F. J. Elliott, S. Legvold et F. H. Spedding: Phys. Rev., 94, 1143 (1954), 
appartient à la même famille antiferromagnétique que $\mathrm{FeCl}_{2}$ et $\mathrm{MnAu}_{2}$ et que l'existence d'un champ seuil paraît ainsi parfaitement normale.

Quant à la transition vers un ferromagnétisme qui se produit quand la température s'abaisse en dessous de $100^{\circ} \mathrm{K}$, nous proposons de l'attribuer à la variation thermique des constantes d'anisotropie $K_{\mathbf{0}}$ et $K_{1}$. Le fait que la discontinuité d'aimantation reste extrêmement importante au voisinage du point de transition $T_{u}$ montre que la transition appartient au premier type.

Pour appuyer cette interprétation, nous avons déterminé $H_{s}$ et $M$ d'après les données expérimentales et représenté $H_{s} / M$ en fonction de la température (Fig. 3). Nous obtenons ainsi une courbe qui

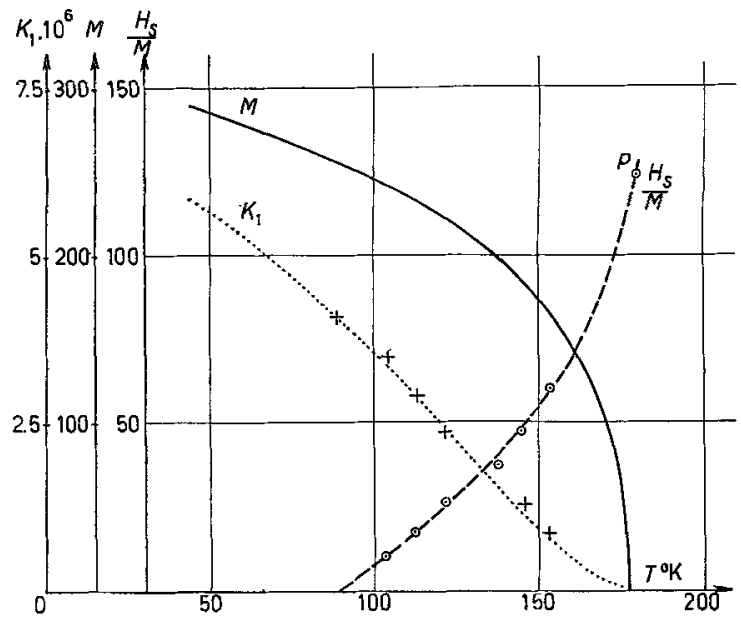

Fig. 3. coupe l'axe des abscisses à $90^{\circ} \mathrm{K}$ au voisinage immédiat du point $T_{a}$ estimé plus haut être voisin de $100^{\circ} \mathrm{K}$. D'autre part, cette même courbe extrapolée vers $T=T_{n}$ donne une valeur très voisine de $125=n / 2$, conformément aux prévisions théoriques relatives au premier type de transition. Ces faits apportent une preuve décisive en faveur de l'interprétation que nous proposons.

Au contraire une interprétation basée sur la variation thermique des champs moléculaires se heurte, d'une part à l'objection qu'elle n'explique pas la reJation étroite révélée par l'expérience entre le champ seuil et la température $T_{a}$ de transition et d'autre part au fait que la constante de Curie spécifique, dans l'état paramagnétique, possède une valeur pratiquement égale à la valeur théorique correspondant à $\mathrm{Dy}^{+++}$, ce qui montre que la variation thermique des coefficients de champ moléculaire est très faible, comme nous l'avons rappelé plus haut.

\section{6. - Valeur de la constante d'anisotropie $K_{1}$.}

De la valeur du champ seuil $H_{s}$, connaissant d'autre part $n$ et $M$, il est possible d'en déduire $r-r^{\prime}$, puis $K_{1}$. Ces valeurs ont été reportées sur la Fig. 3 en fonction de $T$. On trouve ainsi qu'à $90^{\circ} \mathrm{K}$, la constante $K_{1}$ est voisine de $4 \cdot 10^{6} \mathrm{erg} / \mathrm{g}$, soit environ $6.5 \cdot 10^{8} \mathrm{erg} / \mathrm{mole}$. Cette valeur est à rap- 
procher de la valeur de la constante d'anisotropie du composé $\mathrm{MnBi}$, égale à $3.5 \cdot 10^{8} \mathrm{erg} / \mathrm{mole}$. Ainsi les constantes d'anisotropie qu'il est nécessaire d'introduire dans notre système d'interprétation sont du même ordre de grandeur que celles de corps ferromagnétiques connus.

La susceptibilité initiale moyenne $s_{m}$ décrô̂t de $4 \cdot 10^{-3}$ à $2.8 \cdot 10^{-3}$ quand la température s'abaisse de $178^{\circ} \mathrm{K}$ à $168^{\circ} \mathrm{K}$. Cette décroissance est attribuable à la diminution de $s_{p}$ et de $s_{n}$. La susceptibilité parallèle $s_{p}$ est donnée par l'équation (8); à $10^{\circ}$ au-dessous $T_{n}$, de on trouve que $(M / T) \mathrm{d} T / \mathrm{d} M$ est approximativement égal à -0.13 , d'où finalement $s_{p}=1.9 \cdot 10^{-3}$ soit sesinblement la moitié de la valeur correspondant à $T=T_{n}$. La croissance de $r^{\prime}$ à mesure que l'on s'écarte de $T_{n}$ produit une diminution de $s_{n}=1 / n\left(1+r^{\prime}\right)$. L'expérience ne donne que $r-r^{\prime}$ qui est égal à 0.30 à $10^{\circ}$ au-dessous de $T_{n}$. Si on pose $r^{\prime}=k r$, il faut donner à $k$ la valeur raisonnable de 0.43 pour obtenir finalement une valeur de $s_{m}$ égale à la valeur expérimentale (en négligeant l'effet de la croissance de $r^{\prime}$ sur $s_{p}$ ).

En admettant maintenant que $k$ conserve la valeur 0.43 quand la température varie, on en déduit que $r+r^{\prime}$ prend la valeur 1 pour $T=T_{b}=160^{\circ} \mathrm{K}$, ce qui donne la température $T_{b}$ à laquelle se produit le passage du point représentatif de la région (II) à la région (III): les valeurs du champ seuil que nous avons utilisées correspondent à des températures inférieures, c'est-à-dire à la région (II) comme nous l'avions admis implicitement.

\section{7. - Application à l'erbium.}

Les propriétés magnétiques de l'erbium, bien que connues avec moins de précision $\left.{ }^{15}\right)$, sont analogues à eelles du dysprosium: on y distingue les mêmes trois régions $A, B$ et $C$.

Dans la région $C$, à $T_{n}=76^{\circ} \mathrm{K}$ environ, apparaît un maximum de susceptibilité égal à $2.4 \cdot 10^{-3}$ : il correspond à $n=420$. Au-dessus de $T_{n}$, on a affaire à un paramagnétisme de Curie-Weiss caractérisé par un point de Curie paramagnétique $\theta_{p}=+40^{\circ} \mathrm{K}$ et une constante de Curie spécifique voisine de $C=0.069$, valeur théorique. On obtient ainsi $n^{\prime}-n=2 \theta_{p} / C=1160$, soit $n^{\prime}=1580$. Le rapport $n^{\prime} / n=3.8$ est bien plus petit que dans le eas $d u$ dysprosium.

Dans la région $B$ apparâ̂t un champ seuil qui s'annule vers $30^{\circ} \mathrm{K}$. Le rapport $H_{s} / M$ croît à peu près linéairement en fonction de la température et s'extrapole pour $T=T_{n}$ vers la valeur 190, fort proche, compte-tenu de grosses incertitudes expérimentales, de la valeur théorique $\frac{1}{2} n=210$. A $30^{\circ} \mathrm{K}$, la

(15) F. J. Elitiott, S. Legvold et F. H. Seedding: Phys. Rev., 100, 1595 (1955). 
constante d'anisotropie $K_{1}$, calculée avec la même méthode que celle du dysprosium, est voisine de $6 \cdot 10^{6} \mathrm{erg} / \mathrm{g}$ ou $10^{9} \mathrm{erg} / \mathrm{mole}$.

\section{8. - Conclusions.}

En résumé, l'expérience permet de distinguer parmi les substances antiferromagnétiques une catégorie bien individualisée correspondant à des valeurs. positives de $n^{\prime}$, e'est-à-dire à des interactions positives à l'intérieur de chaque sous-réseau et à des valeurs de $n$ petites devant $n^{\prime}$. Ces conditions favorisent. l'observation du champ seuil $\left(\mathrm{FeCl}_{2}, \mathrm{MnAu}_{2}, \ldots\right)$ et éventuellement le passage de l'état antiferromagnétique à l'état ferromagnétique sous l'influence de la. variation thermique des constantes d'anisotropie $K_{0}$ et $K_{1}$.

Les mêmes conditions favorisent également la présence d'un moment magnétique permanent superficiel, lié aux parois de Bloch séparant les domaines. élémentaires antiferromagnétiques, comme nous l'avons montré antérieurement $\left({ }^{6}\right)$.

Il semble done justifié de ranger ces substances dans une catégorie spéciale: celle des substances métamagnétiques, reprenant ainsi une vieille dénomination forgée par BECQUEREL ( $\left.{ }^{1}\right)$ pour certaines d'entre elles $\left(\mathrm{CoCl}_{2}\right.$, $\left.\mathrm{NiCl}_{2}, \ldots\right)$, avant que la signification de leurs assez étranges propriétés magnétiques ait été élucidée.

Il faut toutefois s'attendre à ce que, dans le domaine des très basses températures, la théorie exposée ci-dessus ne suffise pas à rendre compte de toutes les propriétés magnétiques de la substance, notamment quand les porteurs de magnétisme possèdent un moment orbital. Il faut alors tenir compte de la. décomposition des niveaux magnétiques dans le champ électrique cristallin. Si l'écartement de ces niveaux est de l'ordre de $n M^{2}$ ou de $n^{\prime} M^{2}$, les phénomènes deviennent extrêmement compliqués et ne peuvent plus s'interpréter d'une manière intuitive. 